\title{
On the Effectiveness of Logical Device Aggregation in Multi-radio Multi-hop Networks
}

\author{
Michele Rossi ${ }^{* \star \ddagger}$, Leonardo Badia ${ }^{\star \ddagger}$, Paolo Giacon ${ }^{\star}$, Michele Zorzi ${ }^{\dagger \ddagger}$
}

\begin{abstract}
In this paper we investigate new features of future generation networks, where the availability of several different access technique is integrated by means of common radio resource management interfaces and, at the same time, information about the mobility context and the physical proximity of some nodes is exploited. In particular, we advocate to utilize this knowledge in order to create routing groups of adjacent nodes, which might be beneficial in order to improve connectivity, decrease signalling overhead and increase transmission efficiency. An analytical framework is proposed, which allows the performance evaluation of device aggregation algorithms, by measuring several connectivity metrics (routing hierarchy, amount of information to be exchanged, energy consumption) under the cases where either routing groups are established or not. In this way, many detailed insights for the network connectivity performance are obtained, which can be adapted to any context due to the analytical formulation. Therefore, it might be useful for the evaluation of the grouping effectiveness and its application to real cases.
\end{abstract}

\section{INTRODUCTION}

Multiple radio multiple access scenarios is a key issue for current and future generation wireless networks. The tremendous advancements achieved in the last few years in the wireless technology world have made it possible to integrate different radios in single portable devices, thereby opening up new marketing opportunities as well as new technological solutions. Clearly, these new systems pose new challenges for both network operators and protocol designers whose goal is now to provide efficient mechanisms to let such complex networks cooperate and possibly promote device aggregation (and resources distribution) in an efficient manner, so as to take advantage of the diversity introduced by the presence of multiple radio interfaces [1] ("technology diversity"). These topics are currently the object of international projects. Among others, we cite here the European Ambient Network project [2]. For instance, a possible challenge to be solved is represented by the problem of connecting every user with the "best" in range technology, at every time. In fact, the presence of multiple technologies has the potential to allow for an increased performance. For instance, in the presence of multiple

*Corresponding author, e-mail: mrossi@ing.unife.it. ${ }^{\star}$ Department of Engineering, University of Ferrara, via Saragat 1, 44100 Ferrara, Italy. ${ }^{\dagger}$ Department of Information Engineering, University of Padova, via Gradenigo 6B, 35131 Padova, Italy. ${ }^{\ddagger}$ Consorzio Ferrara Ricerche (CFR), via Saragat 1, 44100 Ferrara, Italy. access technologies the system coverage, and hence the terminal reachability, may be extended with respect to the single technology case. Furthermore, devices may decide, in either a coordinated or completely uncoordinated fashion, to switch to less congested systems, thereby achieving load balancing with a subsequent benefit in terms of perceived performance and overall network utilization. However, these are just examples of the many issues that have to be solved in such a kind of networks. In this paper, we focus on the reachability issue, where we are interested in understanding whether it is worth to perform logical device aggregation (or "grouping"). Many grouping approaches have been proposed in the literature so far [3][5], where the clustering of network devices was used to improve routing as well as MAC [6]. However, all these contributions focused on a single technology environment. Our study here is considerably different as we add a new and important dimension to the device aggregation. In fact, we allow different technologies to coexist at both access points (APs) and devices. Our study strives from the practical observation that mobile users often tend to move in aggregation, i.e., according to the so called group mobility behaviors [7]. Examples of group mobility might be found in our daily life, e.g., in a group of people in the same vehicle (car, shuttle, train, etc.) or pursuing a common task within the same geographical area (rescue squads, groups of tourists moving within a museum, etc.). In these cases, it might be beneficial for the users to perform logical device aggregation and elect leaders in charge of coordinating the transmissions within each group. Grouping, in some cases, may increase efficiency as well as reachability of the terminals. For instance, the efficiency may be increased as the transmissions within every group may be handled locally by the group leader, thereby allowing for more efficient forwarding strategies. Our goal here is to derive an analytical model in order to capture the essential properties of such a kind of network in order to assess the possible benefits of device aggregation. The remainder of this paper is organized as follows. First of all, in Section III we present the models that we propose to represent physical/transmission aspects such as user positions, radio interface distributions, propagation model and transmission powers. In Section IV, we characterize the routing group (RG) size as a function of various system parameters. Such a characterization is pivotal for 
all the following analytical derivations. In Section V, we briefly describe how algorithms for routing group operate and we subsequently find the average energy spent for maintaining RG structures. In Section VI, we focus on the analysis of the energy required to transmit to all users in the network with and without grouping. Based on our analytical framework, in Section VII we present some results that highlight the worthiness of grouping users in terms of improved reachability of the terminals. Finally, in Section VIII we report the conclusions of our work.

\section{Heterogeneous Networks AND Routing GROUPS}

In this work, we address heterogeneous networks where users and access points (APs) possess multiple radio interfaces and operate within the same geographical area. In such an environment, it might be beneficial to join all or part of the users in what we refer here to as routing groups (RGs). This logical grouping is performed with the aim of taking advantage of the users' physical proximity and possibly of similar mobility patterns in order to improve the efficiency in transmitting data and/or handling network related procedures such as the handover between different APs. As an example, multiple users moving together and handing over at the same time between the same pair of access points may be joined in a routing group so that a single message (to the RG leader) needs to be exchanged to successfully accomplish to the handover procedure, instead of using one dedicated channel (an unicast message) for every user. This is, in general, true every time the information can be shared among users, that is, for all applications where a sort of multicast messaging is inherently supported. In other cases, we may join users according to their access technologies and "cluster" them to increase the transmission efficiency. Think again, for instance, to a vehicle occupied by several passengers, which henceforth move with a similar pattern. In such a case, it could be efficient to elect a RG leader, e.g., the on-board multimedia system, and transmit the information related to, e.g., close tourist attractions, route information, tv programs, to all users in the vehicle in a multicast fashion. In such a case, the RG leader will retrieve the wanted information from the external network through dedicated access points and then, the information could be more efficiently distributed to the RG members by exploiting their physical proximity. This simple example illustrates the opportunities and advantages offered by a grouping of network entities when they exhibit a group mobility behavior.

In this work, instead of deriving specific algorithms for handling and creating RGs, we focus on the effectiveness of the grouping principle as a function of the node and $\mathrm{RG}$ leaders densities, and of the number and type of radio interfaces owned by the users. In particular, our aim is to quantify such benefits and weigh them against the costs incurred in creating and maintaining RG structures.

\section{SySTEM MODEL}

We consider an heterogeneous network where a number of access points (APs) and a number of users coexists. Both APs and users support a number of different radio technologies which can be described by the indices $1,2, \ldots, J$, where technologies are indicated with integers numbers and sorted according to the required transmission energies. That is, $E_{i}^{t x} \leq E_{j}^{t x}$ iff $i<j$. Accordingly, we define three vectors $\mathbf{E}^{t x}=\left\{E_{1}^{t x}, E_{2}^{t x}, \ldots, E_{J}^{t x}\right\}, \mathbf{E}^{r x}=$ $\left\{E_{1}^{r x}, E_{2}^{r x}, \ldots, E_{J}^{r x}\right\}$ and $\mathbf{r}=\left\{r_{1}, r_{2}, \ldots, r_{J}\right\}$ tracking the energies required to transmit and receive a single bit and the maximum transmission ranges for every technology, respectively. Not all nodes offer all radio interfaces and, in general, the available interfaces may differ between different nodes. Here, we assume that a generic node has an interface of type $j$ with a given probability $p_{j}$ and that interfaces are assigned independently to each user in the network. For the topology, we consider that users are independently placed according to a planar Poisson process of intensity $\rho$ [8], i.e., the average number of users within an area $\mathcal{A}$ is given by $\rho \mathcal{A}$, whereas the probability to have exactly $n$ nodes in this area is derived as $\mathcal{P}(n, \mathcal{A})=$ $\left((\rho \mathcal{A})^{n} / n !\right) \exp (-\rho \mathcal{A})$.

At the physical layer, every transceiver device has a given receiver sensitivity $\eta_{j}$ which depends on the considered radio interface $j \in\{1,2, \ldots, J\}$. We assume that packets are correctly decoded when the received power is above the respective technology-dependent sensitivity threshold. According to the analysis presented in [9], the propagation loss $L(d)$ (in decibel) at a distance $d$ is given by $L(d)=K_{0}+K_{1} \ln d+s$, where $K_{0}$ and $K_{1}$ are proper constants, while $s$ is a shadowing sample which is assumed to be log-normally distributed with zero mean and standard deviation $\sigma_{\text {shad }}$. Thus, the received power (decibel) at the generic interface $j$ of a given node is $P_{r x, j}(d)=P_{t x, j}-L(d)$, where $d$ is the distance between the source (S) and the node itself and $P_{t x, i}$ is the power used by $\mathrm{S}$ to transmit. We say that a packet transmitted with technology $j$ is correctly received if $P_{r x, j}(d) \geq$ $\eta_{j}$. Observe that, as the channel attenuation is modeled accounting for a log-normal shadowing contribution, the received power (and hence the correctness of a packet transmission) as a function of the distance is a random variable depending on the shadowing pdf. Now, if we refer to a probabilistic threshold $p_{c} \in(0,1)$, we can define the maximum transmission range $r_{j}$ for a given technology $j \in\{1,2, \ldots, J\}$ as the maximum distance $d_{\text {max }, j}$ for which the relation $\operatorname{Prob}\left\{P_{r x, j}(d) \geq \eta_{j}\right\}=p_{c}$ holds. Then, we can set $r_{j}=d_{\max , j}$ by observing that $r_{j}$ is conditioned on the quality of service (QoS) level captured by the probability $p_{c}$. Hence, by repeating the above reasonings for every technology, it is possible to derive the maximum transmission ranges vector $\mathbf{r}$ as a function of the transmission power levels $P_{t x, j}$ and of the radio sensitivities $\eta_{j}$, where $\mathbf{r}$ is conditioned on the 


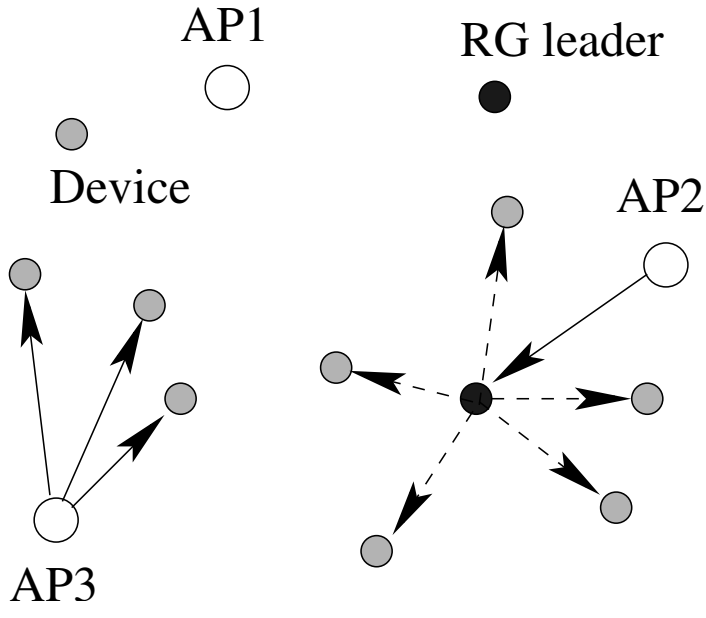

Fig. 1. Considered network architecture.

minimum QoS guarantee $p_{c}$, as explained above. That is, given the QoS requirements, we can always get to a related vector of maximum transmission distances. The analytical formulation that we present in the sequel will make a direct use of the vector $\mathbf{r}$ by, for the sake of simplicity, omitting this last passage but by bearing in mind how maximum ranges are actually derived.

Given the network topology and the radio interface models, we can easily find the density $\rho_{j}$ of nodes with an interface of type $j$. Formally

$$
\rho_{j}=\frac{\sum_{n=0}^{\infty} \mathcal{P}(n, \mathcal{A}) \sum_{k=0}^{n} k P_{j}(k \mid n)}{\mathcal{A}}=p_{j} \rho
$$

where $\mathcal{A} \in \mathbb{R}^{+}, P_{j}(k \mid n)=\left(\begin{array}{l}n \\ k\end{array}\right) p_{j}^{k}\left(1-p_{j}\right)^{n-k}$. Of course, $\sum_{j=1}^{J} \rho_{j}$ may also be larger than $\rho$.

In Fig. 1, we report an illustrative example of the considered network architecture. Both nodes and access points (APs) are randomly placed according to a planar Poisson distribution, as introduced above. APs are assumed to possess all the available technologies and are therefore able to communicate with every in range device. Network devices are classified in two different categories: regular devices (referred to as Device in the figure) and routing group leaders (referred to as $R G$ leaders). RG leaders are also assumed to have all technologies, whereas regular devices own technology $j \in\{1,2, \ldots, J\}$ with probability $p_{j}$. As reported in the figure, we account for two different communication paradigms: in the first case (e.g. AP3) nodes communicate directly with the closest AP, whereas in the second case (e.g. AP2 in the figure) nodes communicate with their RG leader which acts as a relay node for every device in its routing group (RG). The aim of the following analysis is to compare these two possibilities in terms of energy consumption as well as network connectivity.

\section{Calculation of the Average Routing Group SizE AND NUMBER OF MEMBERS}

In this section we characterize the RG structure, by analyzing the RG geographical extension and number of nodes. These results will be used in the following sections for the calculation of the average energy required to deliver data when RGs structures are in place. We consider that RG leaders are uniformly distributed within the network and that every node is elected as a leader with a probability $p_{L D}$ which is the same for all nodes [9]. We note that depending on the specific RG scheme at play, the average RG size may vary, as for standard clustering approaches [10], [11]. Hence, we can choose $p_{L D}$ to reflect, in a very simple manner, the average size of the formed RGs and hence to account for the specific RG formation algorithm at play. For what concerns the node positioning, we still consider all devices (standard nodes and RG leaders) to be placed according to a planar Poisson distribution. Now, we focus on a given node and we assume that the node was elected as a RG leader. Then, starting from this leader, we seek for the 1 st, 2 nd, ..., $n$th device surrounding it, where the 1 st node is the closest to the leader, the 2 nd is the second closest and so on. Moreover, we refer to $d_{1}, d_{2}, \ldots, d_{n}$ as the random positions of these $n$ nodes. The joint probability of these positions was first derived in [12] and is given by $P_{n}\left(d_{1}, d_{2}, \ldots, d_{n}\right)=(2 \lambda)^{n} e^{-\lambda d_{n}^{2}} d_{1} \mathrm{~d} d_{1} d_{2} \mathrm{~d} d_{2} \cdots d_{n} \mathrm{~d} d_{n}$

where $\lambda=\pi \rho$ and $0 \leq d_{1} \leq d_{2} \leq \cdots \leq d_{n}$. The absolute probability that the $n$th nearest neighbor is distant $d_{n}$ from the RG leader is obtained by integrating Eq. (2) with respect to $d_{1}$ from 0 to $d_{2}$, with respect to $d_{2}$ from 0 to $d_{3}, \ldots$, with respect to $d_{n-1}$ from 0 to $d_{n}$ and is given by

$$
P_{1}\left(d_{n}\right)=\frac{2 \lambda^{n} e^{-\lambda d_{n}^{2}} d_{n}^{2 n-1}}{(n-1) !}
$$

Moreover, the probability that the closest leader is the $n$th nearest node is given by

$$
P \text { (node } n \text { is the closest leader })=\left(1-p_{L D}\right)^{n-1} p_{L D} \text {. }
$$

The joint pdf that the $n$-th closest node is the closest leader and its position is $d_{n}$ is then given by the product of Eqs. (2) and (3) as follows ${ }^{1}$

$P\left\{n\right.$th is leader, $\left.d_{n}\right\}=\frac{2 \lambda^{n} e^{-\lambda d_{n}^{2}} d_{n}^{2 n-1}\left(1-p_{L D}\right)^{n-1} p_{L D}}{(n-1) !}$

The marginal pdf $P\left\{d_{n}\right\}$ is therefore found as

$$
\begin{aligned}
P\left\{d_{n}\right\} & =\sum_{n=1}^{\infty} P\left\{n \text {th is leader, } d_{n}\right\} \\
& =\beta \sum_{n=1}^{\infty} \frac{\alpha^{n}}{(n-1) !}=\beta \alpha e^{\alpha}
\end{aligned}
$$

${ }^{1}$ In fact, we assume here that leaders are elected independently of their geographical positions. 


$$
\begin{aligned}
\bar{E}_{R G}(\mathcal{A}, T=\xi \Delta T) & =\sum_{j=1}^{J} \sum_{n=1}^{\infty} \mathcal{P}(n, \mathcal{A}) \sum_{k=1}^{n}\left\{k P_{j}(k \mid n) b_{j} n_{j}^{h}\left[E_{j}^{t x}+E_{j}^{r x} \varepsilon_{j}\right]\right\} \\
\varepsilon_{j} & =\sum_{n=2}^{\infty} \mathcal{P}\left(n, \pi \min \left(r_{j}, \bar{r}_{R G}\right)^{2}\right) \sum_{k=1}^{n}(k-1) P_{j}(k \mid n)
\end{aligned}
$$

where $\alpha=\left(1-p_{L D}\right) \lambda d_{n}^{2}$ and $\beta=\left[p_{L D} /(1-\right.$ $\left.\left.p_{L D}\right)\right] d_{n} e^{-\lambda d_{n}^{2}}$. After straightforward calculations, $P\left\{d_{n}\right\}$ can be re-written as

$$
P\left\{d_{n}\right\}=2 \lambda p_{L D} d_{n} e^{-\lambda p_{L D} d_{n}^{2}}
$$

Now, the average closest distance between two leaders can be computed by

$$
\mathbb{E}\left[d_{n}\right]=2 \gamma \int_{0}^{\infty} x^{2} e^{-\gamma x^{2}} \mathrm{~d} x=\frac{1}{2 \sqrt{\rho p_{L D}}}
$$

where $\gamma=\lambda p_{L D}$. From this result, we can calculate the average range $\left(\bar{r}_{R G}\right)$ and the average area $\left(\mathcal{A}_{R G}\right)$ covered by a $\mathrm{RG}$, as $\mathbb{E}\left[d_{n}\right] / 2$ and $\mathcal{A}_{R G}=\pi \bar{r}_{R G}^{2}$, respectively. Therefore

$$
\bar{r}_{R G}=\frac{1}{4 \sqrt{\rho p_{L D}}}
$$

\section{RG Formation Algorithms AND Related ENERGY CONSUMPTION}

Routing groups (RGs) can be usually formed exploiting a distributed approach. That is, users cooperate and exchange data in order to gain information about their physical proximity and, at the same time, to measure the worthiness of grouping with other network entities. This involves the periodical exchange of the so called HELLO messages between mobile nodes. In each HELLO, any given node can include the list of its "stable neighbors", that can be seen as the list of nodes that have been in its close proximity for a long enough period of time [13]. Specific algorithms for the creation of RG structures are not in the scope of the present paper. However, it is easy to understand that if movements are correlated, stable nodes are likely to remain in close proximity of the sending device and are therefore good candidates to be grouped with it. We further assume that a leader is elected within each RG; this device has the special role of handling the data traffic so as to optimize the transmission and the access to the channel of the RG members. This can be seen, as in standard clustering algorithms for ad hoc networks [11], as a way to partially centralize the transmission control thereby enhancing the performance. We assume that every interface $j \in\{1,2, \ldots, J\}$ sends HELLO messages with an interface-specific period $T_{j}$ and we refer to $b_{j}$ as the number of bits composing HELLO packets sent by an interface of type $j$. Moreover, we consider that all $T_{j} \mathrm{~s}$ are multiple of a reference time period $\Delta T$ such that
$T_{j}=\xi_{j} \Delta T, \xi_{j} \in \mathbb{N}^{+}, j \in\{1,2, \ldots, J\}, \Delta T \in \mathbb{R}^{+}$. If we define the least common multiple (LCD) of all $T_{j} \mathrm{~s}$ as $\xi \Delta T$, then we have that

$$
n_{j}^{h}=\frac{\xi}{\xi_{j}}
$$

is the number of HELLOs sent by the $j$-th interface in a time period equal to $\xi \Delta T$. According to the above model and assumptions, the energy spent to maintain the $\mathrm{RG}$ structures over an area $\mathcal{A}$ in a time period of $\xi \Delta T$ seconds can be derived according to Eq. (11) on the top of this page, where $\varepsilon_{j}$ is the mean number of nodes receiving the HELLO message sent by a given sending node and using interface $j$. Here, we reasonably assume that these HELLO packets are only decoded by the node neighbors whose distance is less than or equal to $\bar{r}_{R G}$, i.e., in the worst case RG related information spans over two adjoining RGs. ${ }^{2}$ The energy spent per unit of area and time to create and maintain RG structures is therefore derived as

$$
\bar{E}_{R G}^{*}=\frac{\bar{E}_{R G}(\mathcal{A}, \xi \Delta T)}{\mathcal{A} \xi \Delta T}
$$

We observe that this calculation holds for uniform node and radio interface distributions and for a generic $R G$ grouping algorithms where RGs are formed and maintained in a distributed manner thanks to periodic exchanges of neighbors information. Moreover, Eq. (11) is related to the maintenance phase, whereas the initial transient (discovery) phase, which could be reasonably characterized by a higher energy consumption is neglected as it does not contribute to the steady-state energy metric.

In the following sections, we consider the unicast data transmission case by focusing on both the scenarios where RGs are in place and the one where no RGs are accounted for. Observe that, in the former case unicast flows are routed first from the closest AP to the RG leader (AP $\rightsquigarrow$ $\mathrm{RG}$ leader) and then optimally and locally distributed to the RG members (RG leader $\rightsquigarrow R G$ members). In the latter scenario (no RGs), instead, unicast flows are transmitted directly by the APs to every device in the network. See Fig. 1 for an example of the two above cases, where the former is illustrated by AP3, whereas the latter is represented by the transmission originated from AP2, respectively.

${ }^{2}$ Devices may decide, based on the RG membership information contained into the HELLO packet header, whether they should decode or ignore the packet (thereby saving energy). This mechanism could be implemented through special header tags as done, e.g., in the Bluetooth system [14] to discriminate packets belonging to different piconets. 


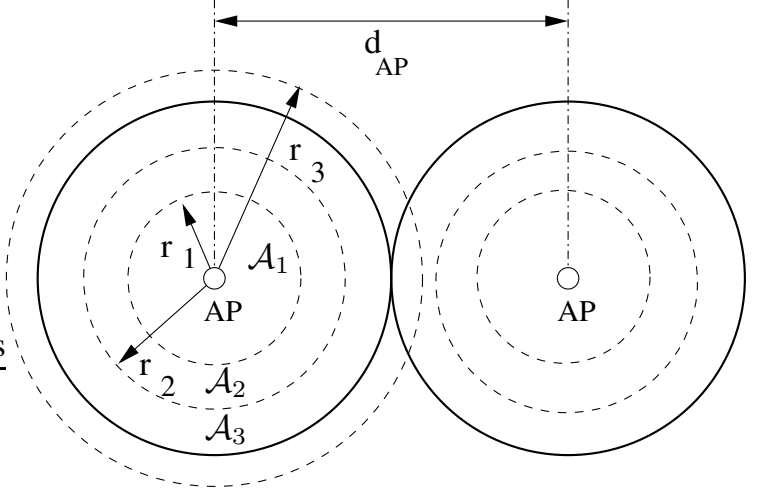

Fig. 2. Illustration of the AP coverage capabilities and their relation to the radio technologies transmission ranges.

\section{ENERGY CONSUMPTION FOR THE DELIVERY OF UNICAST DATA}

In this section we consider the delivery of unicast traffic to a set of users surrounding a given AP. Moreover, we consider the worst case where each user requires the unicast flow and all flows have the same bit-rate $B_{U}$. These two assumptions can be seen as the situation where all nodes in the network are active and the common bit-rate can be roughly interpreted as the average transmission rate delivered to the end users. The aim of the following analysis is to characterize the energy spent per unit of area and time in transmitting these flows to all users in the network. We further consider that access points are placed according to a uniform distribution with density $\rho_{A P}$ and that are equipped with all the technologies present in the network. The average distance between two APs is therefore given by $\bar{d}_{A P}=1 /\left(2 \sqrt{\rho_{A P}}\right)\left(\right.$ Eq. $(8)$ with $\left.p_{L D}=1\right)$. Hence, on average each AP is in charge of delivering data to all users placed within a circle of radius $\bar{r}_{A P}=\bar{d}_{A P} / 2$. To help understanding the following analysis, in Fig. 2 we report a scheme depicting two neighboring APs and the radio technologies transmission ranges (vector $\mathbf{r}$ ) in a scenario with $J=4$ different radio technologies. ${ }^{3}$ As clearly shown in the figure, on average each AP only needs to serve the area covered by technology $i$ for which $r_{i-1}<\bar{r}_{A P}$ $\left(r_{0}=0\right)$. For instance, in Fig. 2 region 3 is the last one that needs to be considered by a given AP to cover all its users; in fact, $r_{3}>r_{A P}$. We refer to the number associated with the last region as $I \leq J$. More precisely, transmission ranges $r_{i}, i \in\{1,2, \ldots, \bar{I}\}$ form $I$ circular anulii with area $\mathcal{A}_{i}=\pi\left[\min \left(r_{i}, \bar{r}_{A P}\right)^{2}-\min \left(r_{i-1}, \bar{r}_{A P}\right)^{2}\right]$ with $r_{0}=0$ and $i \in\{1,2, \ldots, I\}$. The density of nodes with technology $j$ is still given by $\rho_{i}$ as derived in Eq. (1). The average number of users that have to be reached in region $i, \bar{n}_{i}$, is found

\footnotetext{
${ }^{3}$ The 4 th technology is not shown in the figure but we assume to have $r_{4}>r_{3}$.
}

according to

$$
\bar{n}_{i}= \begin{cases}\rho \pi\left[\bar{r}_{A P}^{2}-r_{J-1}^{2}\right] & i=J \text { and } r_{i}<\bar{r}_{A P} \\ \rho \mathcal{A}_{i} & \text { otherwise }\end{cases}
$$

where the first line in the above equation accounts for the case where technology $J$ can not completely cover the serving area assigned to the AP (that is $r_{J}<r_{A P}$ ). If this occurs, part of the AP serving area $\left(\pi\left[\bar{r}_{A P}^{2}-r_{J}^{2}\right]\right)$ remains uncovered and the total number of user to be served in region $i=J\left(\bar{n}_{J}\right)$ is actually higher than the number of users reachable with the highest rank technology $J$.

\section{A. Case without RGs}

The aim of this section is to compute the average energy required to deliver the unicast flows to all users served by a given AP. We start with region 1 , where users are reachable with every technology $\left(r_{j} \geq r_{0}\right.$, $\forall j \in\{1,2, \ldots, J\})$. Moreover, we assume that the AP has a complete knowledge regarding the users to be served and can therefore optimize its transmission energies as follows. First of all, the AP serves all users in $\mathcal{A}_{1}$ having technology 1 , hence $\bar{n}_{1,1}=\rho_{1} \mathcal{A}_{1}$ users are served (on average) first by exploiting $\bar{n}_{1,1}$ unicast channels, where we refer to $\bar{n}_{i, j}$ as the average number of users served in region $i$ by technology $j$ (whose region is $\mathcal{A}_{j}$ ). For what concerns technology 2 , the AP on average needs to serve $\bar{n}_{2,1}=\min \left(\bar{n}_{1}\{2\right.$ and no 1$\left.\}, \bar{n}_{1}-\bar{n}_{1,1}\right)$ users in region 1 , where $\bar{n}_{1}\{2$ and no 1$\}$ is the average number of users in region 1 that have technology 2 but do not have technology 1 , whereas $\bar{n}_{1}$ is the total number of users in region 1 . The probability of having $k$ users over $n \geq k$ with interfaces $j \in\{2, \ldots, J\}$ and without interfaces $1,2, \ldots, j-1$ is found as

$$
P_{\{j \text { and no } 1,2, \ldots, j-1\}}(k \mid n)=\left(\begin{array}{l}
n \\
k
\end{array}\right)\left(\tilde{p}_{j}\right)^{k}\left(1-\tilde{p}_{j}\right)^{n-k}
$$

where $\tilde{p}_{j}=p_{j}\left[\prod_{m=1}^{j-1}\left(1-p_{m}\right)\right]$, and $p_{j}$ is the probability for a generic user of having interface of type $j$. The average number of users $\bar{n}_{i}\{j$ and no $1,2, \ldots, j-1\}$ in region $i$ with interfaces $j>1$ and without interfaces of type $1,2, \ldots, j-1$ is therefore found by averaging $p\{j$ and no $1,2, \ldots, j-1\}$ over $n$, accounting for the Poisson distribution and the area $\mathcal{A}_{i}$. Hence

$$
\bar{n}_{i}\{j \text { and no } 1,2, \ldots, j-1\}=\rho p_{j}^{1} \mathcal{A}_{i}
$$

In general, the quantities $\bar{n}_{i, j}$ are obtained according to Eq. (16) on the top of the next page, where $i \in$ $\{1,2, \ldots, I\}$ and $j \in\{1,2, \ldots, J\}$. The total energy expenditure per unit area and time is therefore found as

$$
\bar{E}_{U N I-n o R G}=\frac{\sum_{i=1}^{I} \sum_{j=i}^{J} \bar{n}_{i, j}\left(E_{j}^{t x}+E_{j}^{r x}\right) B_{U}}{\mathcal{A}_{A P}}
$$

where $\mathcal{A}_{A P}=\pi \bar{r}_{A P}^{2}$ is the area served, on average, by a single AP. Besides the energy expenditure, another 


$$
\bar{n}_{i, j}= \begin{cases}0 & j<i \\ \rho_{1} \mathcal{A}_{i} & j \geq i \text { and } j=1 \\ \min \left(\bar{n}_{i}\{j \text { and no } 1,2, \ldots, j-1\}, \bar{n}_{i}-\sum_{k=1}^{j-1} \bar{n}_{i, k}\right) & j \geq i \text { and } j>1\end{cases}
$$

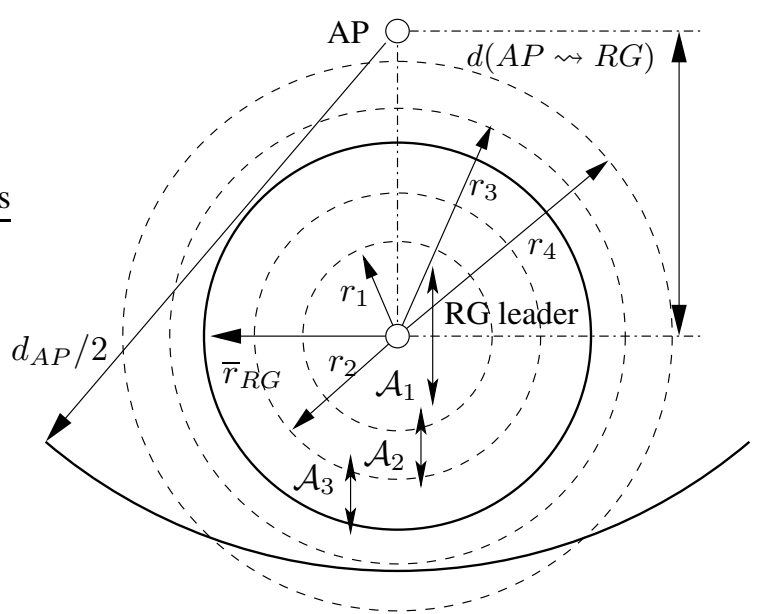

Fig. 3. Diagram for the calculation of the energy spent in transmitting unicast traffic in the RG case.

interesting performance metric to look at is the average number of uncovered users. These are users that do not own all interfaces and that are not reachable by any AP; their average number $\bar{n}_{u}$ is promptly obtained as

$$
\bar{n}_{u}=\sum_{i=1}^{I} \bar{n}_{i}-\sum_{i=1}^{I} \sum_{j=i}^{J} \bar{n}_{i, j}
$$

where $\bar{n}_{i}$ are derived using Eq. (13) and therefore also account for completely uncovered regions (when present). The number of uncovered users per unit area is finally given by $\bar{n}_{u} / \mathcal{A}_{A P}$.

\section{B. Case with RGs}

In this section we consider the scenario where the unicast traffic has to be delivered to all users through dedicated channel and RGs structures are present in the network. In this case, instead of directly transmitting the data traffic to the end users we rely on the presence of RG leaders. As above, we consider a downlink transmission for each data flow, where all flows are assumed to have the same bit-rate $B_{U}$ and one data flow has to be delivered to each user. The diagram for this case is depicted in Fig. 3, where we report an example scenario with $J=4$ radio interfaces. According to the analysis in the previous section, we characterize the AP coverage radius by means of $\bar{r}_{A P}$, whereas the RG area covered (served) by the RG leader is modeled through $\bar{r}_{R G}$ (Eq. (9)). In this case, the unicast flows are first transmitted to the RG leader and then optimally delivered from here to the nodes in the RG coverage area. As a working assumption, we assume that RG leaders have all technologies. In practical RG schemes it is, in fact, reasonable to pick RG leaders among the more capable devices. As above, we subdivide the RG area into $I$ regions (in Fig. $3, I=3$ ) and we calculate the average number of reachable users in a RG as $\bar{n}_{R G}=$ $\sum_{i=1}^{I} \sum_{j=i}^{J} \bar{n}_{i, j}$, where the quantities $\bar{n}_{i, j}$ are evaluated from the analysis illustrated in Section VI-A by substituting $\bar{r}_{A P}$ with $\bar{r}_{R G}$ (see Eq. (9)). Similarly, the average number of uncovered users per unit area is evaluated as $\bar{n}_{u} / \mathcal{A}_{R G}$, where $\mathcal{A}_{R G}=\pi \bar{r}_{R G}^{2}$ and $\bar{n}_{u}$ is obtained as in Eq. (18). In the $\mathrm{RG}$ case the transmission takes place in two different phases, where the first one consists of the transmission from the APs to the RG leaders (AP $\rightsquigarrow R G$ leader) and the second one of the transmission from the RG leaders to the RG members ( $R G$ leader $\rightsquigarrow R G$ members). Moreover, the second phase can be seen as a specialization of the algorithm presented in Section VI-A where the RG size $\left(\bar{r}_{R G}\right)$ is used instead of the AP coverage area $\left(\bar{r}_{A P}\right)$. We refer to this energy contribution as $\bar{E}_{U N I, R G}^{(b)}$. For what concerns the first energy contribution (AP $\rightsquigarrow$ RG leader), we reasonably assume that RG sizes are smaller than the AP coverage area, i.e., that $\bar{r}_{R G}^{2} \ll \bar{r}_{A P}^{2}$. In such a case, it is reasonable to consider the distance between APs and RG leaders as uniformly distributed in $\left[0, \bar{r}_{A P}\right]$ and evaluate the energy consumed per unit time in transmitting to a $\mathrm{RG}$ leader as $\bar{E}_{U N I, R G}^{(a)}=\bar{n}_{R G} B_{U} \bar{E}_{A P \rightsquigarrow R G}$, where $\bar{n}_{R G}$ is the average number of reachable users within a RG, $B_{U}$ is the bandwidth for the unicast traffic and $\bar{E}_{A P \rightsquigarrow R G}$ is the average energy spent to transmit one bit from the AP to the RG leader

$$
\bar{E}_{A P \rightsquigarrow R G}=\int_{0}^{\min \left(\bar{r}_{A P}, r_{J}\right)}\left(\frac{2 x}{\bar{r}_{A P}^{2}}\right) \mathcal{E}(x) \mathrm{d} x
$$

where the $\min (\cdot)$ accounts for the fact that when $r_{J}<\bar{r}_{A P}$ the AP serving area $\left(\bar{r}_{A P}\right)$ can not be completely covered by the access point $\left(r_{J}\right)$ and therefore the farthest RG leader reachable by the AP is placed $\min \left(\bar{r}_{A P}, r_{J}\right)$ meters apart from the AP; $\mathcal{E}(x)$ is a mapping giving the minimum energy that can be used to communicate with a node placed $x$ meters apart

$$
\mathcal{E}(x)=\min _{1 \leq j \leq J}\left\{\left(E_{j}^{t x}+E_{j}^{r x}\right) \text { such that } r_{j} \geq x\right\}
$$

where $\mathcal{E}(x)$ is the minimum transmission energy that can be used to reach a node at distance $x$. The average transmission energy per unit area and time is therefore 


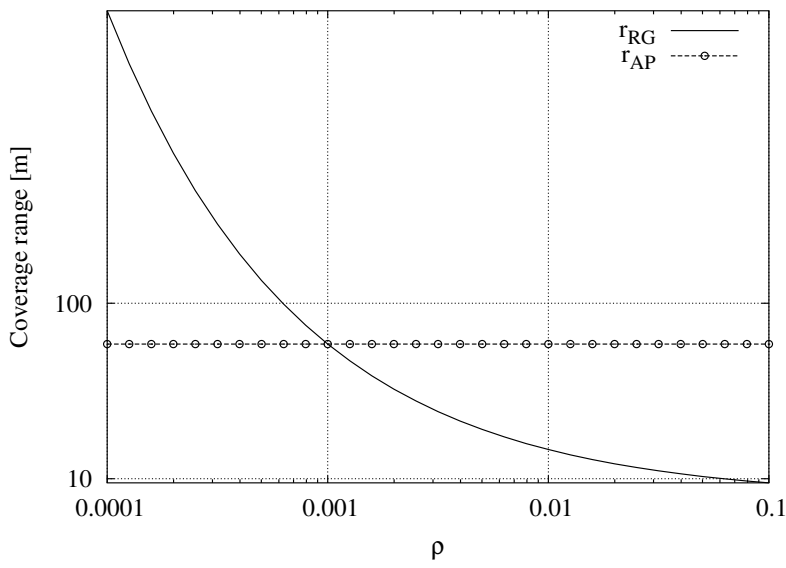

Fig. 4. $\bar{r}_{R G}$ and $\bar{r}_{A P}$ as a function of $\rho$ for $p_{L D}=0.01$ and $\rho_{A P}=$ 0.00001 .

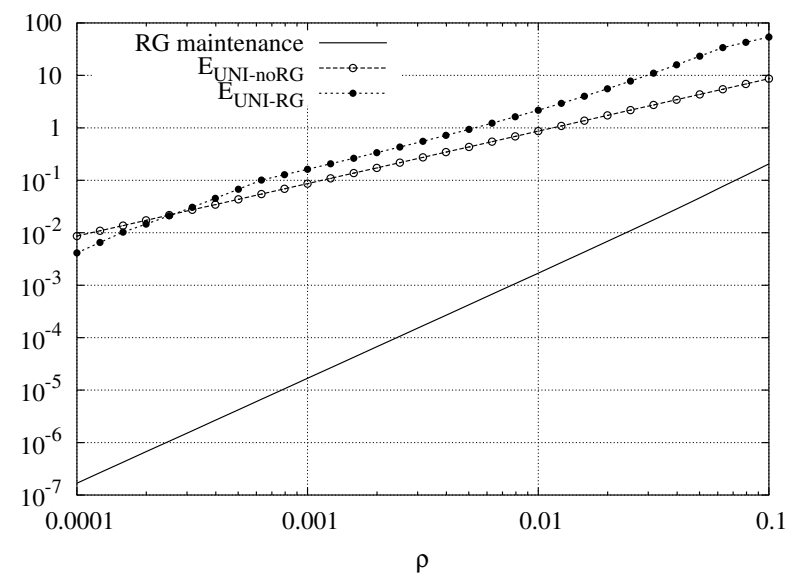

Fig. 5. Total average energy spent per unit area and time.

found as

$$
\bar{E}_{U N I, R G}=\frac{\bar{E}_{U N I, R G}^{(a)}+\bar{E}_{U N I, R G}^{(b)}}{\mathcal{A}_{R G}}
$$

\section{RESULTS}

In this section we report some results for a network case study with $J=3$ technologies, where $\mathbf{E}^{t x}=\{1,2,4\}$, $\mathbf{E}^{r x}=\{1 / 2,3 / 4,2\}, \mathbf{r}=\{10,100,200\}$, where transmission and reception energies are all normalized with respect to $E_{1}^{t x}$, i.e., the transmission energy per bit of the lowest rank technology, whereas transmission ranges $r_{i}$ s are expressed in meters. For what concerns the RG maintenance phase, we assume $\Delta T=1 \mathrm{~s}, \xi_{1}=1, \xi_{2}=2$ and $\xi_{3}=4$ seconds. For the AP density, we assume $\rho_{A P}=10^{-5} \mathrm{~m}^{-2}$ which gives an average inter-AP distance of $d_{A P} \approx 158$ $\mathrm{m}$. We assume that the length of all HELLO messages is 30 bytes. Finally, we consider the following probabilistic distribution of interfaces among users $p_{1}=3 / 4, p_{2}=3 / 4$, $p_{3}=1 / 2$. Given these parameters, we study the impact

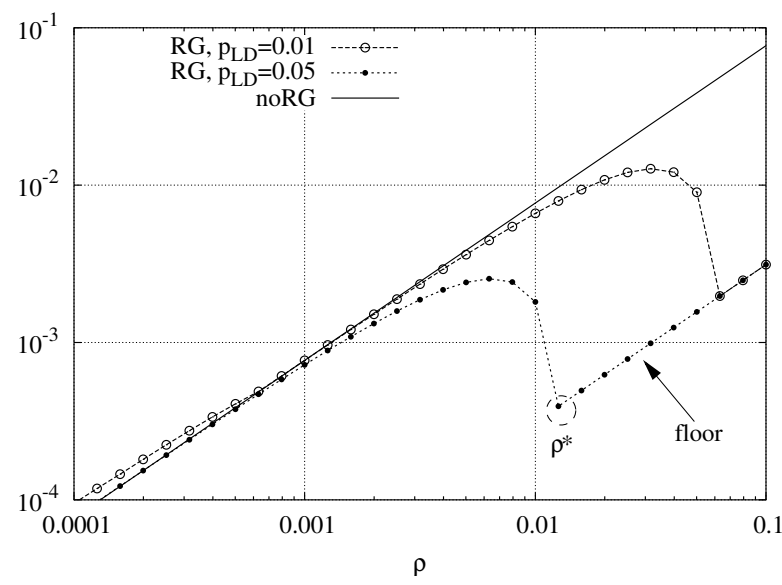

Fig. 6. Average number of uncovered users as a function of the node density $\rho$.

of the node density $\rho$ and of the RG leader probability $p_{L D}$. In Fig. 4, we report $r_{R G}$ and $r_{A P}\left(d_{A P} / 2 \approx 79\right.$ m) as a function of $\rho$ for the above parameters and considering $p_{R G}=0.01$. $r_{A P}$ remains constant (as $\rho_{A P}$ is left unchanged), whereas $r_{R G}$ decreases with an increasing $\rho$. At $\rho=0.001$ the two coverage radii become equal and the density of RG leaders equals $\rho_{A P}$. In Fig. 6 , we focus on the total (transmission/reception/RG maintenance) energy expenditure per unit area and time with and without RGs. For comparison, we also report the energy spent (unit of area and time) to maintain RG structures as a function of $\rho$. This energy contribution is present when RGs are considered, increases with $\rho$ and is significantly lower than the energy needed to transmit to the end users. It shall be observed that this term also highly depends on the vector $\mathbf{E}^{r x}$ and on the HELLO messages transmission periods. Further, as $\rho \rightarrow 1$ its contribution is no more negligible. Therefore, all RG maintenance parameters must be carefully considered for moderate to high densities as their impact on the overall energy balance is relevant. Besides the RG maintenance phase, we observe that the total energy expenditure is higher in the RG case and this is substantially due to the fact that the $R G$ leader acts as a relay by first receiving the data from the APs and then re-transmitting to the RG members. This, from the energy point of view is trivially inefficient and, for this reason, leads to a higher energy expenditure. Nevertheless, we note that the situation may be reversed in the multicast traffic case or when the information to be delivered to the RG member by its own nature can be merged in a single or a reduced number of multicast channels. If this case, grouping and hence relaying packets is expected to lead to considerable benefits in terms of energy. This topic is object of our future research and is not quantitatively addressed here.

In Fig. 6, we report the average number of unconnected users per unit area. These are the users that, on average and 
for the given system parameters, can not be reached by any technology and are therefore disconnected. In the figure we report three case where the first one is for the case without RGs, whereas the remaining two curves are for the RG case with $p_{L D} \in\{0.01,0.05\}$. From this figure it is interesting to observe that RGs and hence the localized presence of RG leaders (or coordinating/relay entities) are actually good for extending the coverage by therefore substantially reducing the probability for a device to be disconnected. In general, the higher $p_{L D}$ the higher the benefits are. We also note that there is a threshold value of $\rho=\rho^{*}$ for which each of the two curves in the RG case reaches a minimum. This density value corresponds to the coverage range $r_{R G}$ for which RGs span over a single region $(I=1)$ and hence all users within a $R G$ are reachable if they have at least one interface. Observe that $\rho^{*}$ depends on both $\rho$ and $p_{L D}$ as $\rho_{R G}=\rho p_{L D}$. For $\rho>\rho^{*}$, the average number of unconnected users still increases with the node density because some users might not have any interface (this occurs with probability $\left(1-p_{1}\right)\left(1-p_{2}\right)\left(1-p_{3}\right)$ ) and these users are the only reason for the floor after $\rho^{*}$. In conclusion, when $\rho_{R G}<\rho_{A P}$ (when $p_{L D}=0.01$, $\rho<0.001$ ) the RG approach leads to both a higher energy consumption and to a higher number of unconnected users. Hence, RGs shall not be activated in such a case. On the other hand, for $\rho_{R G} \geq \rho_{A P}$ RGs introduce (in the unicast case) a higher energy consumption but provide a higher connectivity that, for reasonable values of $\rho$, can exceed the connectivity of the normal operational mode (AP $\rightsquigarrow$ users) by more than one order of magnitude. Finally, it shall be observed that for a given $\rho^{*}$ further increasing $\rho_{R G}$ does not lead to any advantage in terms of connectivity as the difference with respect to the no RG case becomes constant (after $\rho^{*}$ the two curves with and without RGs become parallel). This means that, the choice $\rho_{R G}=\rho_{A P}$ is optimal in the sense that further increasing $\rho_{R G}$, and hence increasing either $\rho$ or $p_{L D}$, does not lead to any improvement in terms of difference in the performance with respect to the no RG case, whereas decreasing it will lead to a smaller difference. Also, since $\rho^{*}$ is reached as soon as all users in a RG are covered by all technologies, given $p_{L D}$ and $r_{1}, \rho^{*}$ is found as $\rho^{*}=1 /\left(4 r_{1} \sqrt{p_{L D}}\right)^{2}$. In fact, for $p_{L D}=0.01$ and $r_{1}=10 \mathrm{~m}, \rho^{*}=0.0625$, in accordance with the results in Fig. 6.

\section{CONCLUSIONS AND FUTURE WORK}

In this paper we focused on next generation wireless network scenarios where both users and access points own multiple radio technologies and can therefore communicate exploiting radio technology diversity. In this context, we introduced the concept of routing group ( $R G$ ) formation as a tool to logically merge users in close proximity and/or moving together. Given the RG concept, we first formulated an analytical framework in order to model the multiradio scenario, by considering uniform and random user placement and a probabilistic radio interface assignment. Subsequently, we investigated the effectiveness of the user aggregation ( $\mathrm{RG}$ ) approach in terms of connectivity, that we expressed here as the density of unconnected users. We found that, under reasonable assumptions, the RG approach has the potential of increasing the connectivity metric of more than one order of magnitude. In our future research, we will extend our analytical framework to more complex scenarios, by also accounting for the multicast traffic case. Also, connection with more elaborate mobility patterns will be sought.

\section{ACKNOWLEDGMENT}

This paper describes work undertaken in the context of the Ambient Network project, which is part of the EU's IST program. In total 41 organizations from Europe, Canada, Australia and Japan are involved in this Integrated Project, which will run from 2004-2005 in its first phase. The views and conclusions contained herein are those of the authors and should not be interpreted as necessarily representing the Ambient Network Project.

\section{REFERENCES}

[1] J. Sachs and H. Wiemann and J. Lundsjö and P. Magnusson, "Integration of multi-radio access in a beyond 3G network," in Proceedings IEEE PIMRC, 2004, pp. 757-762.

[2] "The Ambient Networks Project," http: / / www . ambient-networks.org.

[3] Q. Huang, C. Julien, and G.-C. Roman, "Relying on safe distance to achieve strong partitionable group membership in ad hoc networks," IEEE Transactions on Mobile Computing, vol. 3, no. 2, pp. 192-205, 2004.

[4] P. Mohapatra, C. Gui, and J. Li, "Group communications in mobile ad hoc networks," IEEE Computer Magazine, vol. 37, no. 2, pp. 52-59, 2004.

[5] G.-C. Roman, Q. Huang, and A. Hazemi, "Consistent group membership in ad hoc networks," in Proceedings of ICSE, 2001, pp. 381-388.

[6] Y.-L. Chang and C.-C. Hsu, "Connection-oriented routing in ad hoc networks based on dynamic group infrastructure," in Proceedings ISCC, 2000, pp. 587-592.

[7] M. Zonoozi and P. Dassanayake, "User mobility modeling and characterization of mobility patterns," IEEE Journal on Selected Areas in Communications, vol. 15, pp. 1239-1252, 1997.

[8] D. Stoyan, W. S. Kendall, and J. Mecke, Stochastic Geometry and its Applications, 2nd ed. John Wiley \& Sons, 1995.

[9] D. Dardari, A. Conti, and R. Verdone, "Process estimation through self-organizing collaborative wireless sensor networks," in Proceedings of IEEE Globecom, vol. 4, 2004, pp. 3193-3199.

[10] S. Basagni, "Distributed and mobility-adaptive clustering for multimedia support in multi-hop wireless networks," in Proceedings of IEEE/VTS VTC 1999 Fall, vol. 2, 1999, pp. 889-893.

[11] M. Chatterjee, S. Das, and D. Turgut, "WCA: a weighted clustering algorithm for mobile ad hoc networks," Kluwer Cluster Computing, vol. 5, pp. 193-204, 2002.

[12] H. Thompson, "Distribution of Distance to Nth Neighbour in a Population of Randomly Distributed Individuals," Ecology, vol. 37, no. 2, pp. 391-394, apr 1956

[13] C. Toh, "Associativity-based routing for ad-hoc mobile networks," Kluwer Wireless Personal Communications, vol. 4, pp. 103-139, 1997.

[14] B. A. Miller and C. Bisdikian, Bluetooth revealed: The Insider's Guide to an Open Specification for Global Wireless Communications. Prentice Hall, 2000. 